\title{
LCC from November 2019-February 2020 Lists
}

compiled by Ann Heinrichs

The new classification numbers listed here reflect the most recent information available at the time of publication. Items in this list were selected from 2019 list numbers 11 (November 18) and 12 (December 16) and 2020 list numbers 01 (January 20) and 02 (February 17).

\section{CLASS B}

B132.G63

$$
\begin{aligned}
& \text { Philosophy (General) } \\
& \text { Ancient (600 B.C.-430 A.D.) } \\
& \text { Orient } \\
& \text { By region or country } \\
& \text { India } \\
& \text { Special topics, A-Z } \\
& \text { God }
\end{aligned}
$$

\section{CLASS BL}

BL65.H67

Religions. Mythology. Rationalism

Religion

Religion in relation to other subjects, A-Z

Hospitality

History and principles of religions

European. Occidental

Classical religion and mythology

Special deities and characters of classical mythology, A-Z

BL820.H53

[BL820.L32]

Hero and Leander

Leander and Hero see BL820.H53

Asian. Oriental

By religion

Hinduism

Hindu pantheon. Deities. Mythical characters

Individual deities

Other individual deities, A-Z

BL1225.I65-.I654

BL1225.U33-.U334

Indrān̄i TABLE BL7

Uddhava TABLE BL7

By region or country

China

BL1812.M37

Special topics, A-Z

Mass media 
India

BL2015.D53

Dialogue

Individual religions

Sikhism. Sikh religion Special sects, modifications, etc., A-Z
Mina

BL2018.7.M56

History and principles of religions

African

\section{Ancient Egypt}

Special deities and topics, A-Z

BL2450.H2

$$
\text { Hapi }
$$

By ethnic group, etc., A-Z

BL2480.J65 Jola-Felupe

\section{CLASS BM}

$$
\begin{aligned}
& \text { Judaism } \\
& \text { Sources } \\
& \text { Rabbinical literature } \\
& \text { Special topics, A-Z } \\
& \text { Drinking } \\
& \text { Peace }
\end{aligned}
$$

BM496.9.D7

Judaism

Halakhah. הלכה

General and comparative works on specific areas of the law as defined by Shulhan 'arukh

$$
\begin{aligned}
& \text { Yoreh de‘ah law. יורה דעה } \\
& \text { Special topics, A-Z }
\end{aligned}
$$$$
\text { Special topics, A-Z }
$$

BM523.5.Y37

Practical Judaism

$$
\text { Liturgy and ritual }
$$

Special elements of the liturgy, A-Z

BM670.B43 קריאת שמע על המטה TABLE BM6 Keri’at Shema' ‘al ha-mițah see BM670.B43
Other special topics, A-Z

Odors see BM729.S53

$\begin{array}{lc}\text { [BM729.042] } & \text { Odors see BM729.S53 } \\ \text { BM729.S53 } & \text { Smell CANCEL } \\ \text { BM729.S53 } & \text { Smell. Odors }\end{array}$

CLASS BP

Islam. Bahai Faith. Theosophy, etc.

Islam

Sacred books

Works about the Qur'an

special topics, A-Z

[BP134.E532] Embryology, Human see BP134.H83

BP134.H83 Human embryology

The practice of Islam

Islamic religious life (Descriptive works)

Sufism. Mysticism. Dervishes. صوفية

Monasticism. Sufi orders. Brotherhoods Individual orders, A-Z

BP189.7.S527-.S5272 Sharqāwīyah. شرقاوية TABLE BP3

$\begin{array}{ll}\text { BP190.5.T35 تكفير. } & \text { Topics (not otherwise provided for), A-Z } \\ \text { Takfir. } & \text { Ther }\end{array}$

Bahai Faith

Special topics, A-Z

BP388.V57 Visions

CLASS BQ

Buddhism

Buddhist pantheon

Others, A-Z

$\begin{array}{ll}\text { BQ4890.S55-S554 } & \text { Shers, A-Z } \\ \text { Shinra Myōjin TABLE BQ12 }\end{array}$

Modifications, schools, etc.

Special modifications, sects, etc.

Zen Buddhism

Rinzai

Biography

Founders and other important leaders, A-Z

BQ9399.K64-.K649 
CLASS BR

Christianity
History

$$
\text { By region or country }
$$$$
\text { America }
$$$$
\text { North America }
$$

Canada and British America (General)

BR582.B53 race or ethnic group, A-Z

CLASS BS

The Bible

Texts and versions

Modern texts and versions

English

English versions

Other versions and revisions, A-Z

BS195.R48-R482 Revised New Jerusalem TABLE BS2

Non-European languages
African languages, A-Z
Ewondo TABLE BS5

$\begin{array}{ll}\text { BS325.E96 } & \text { Gungu TABLE BS5 }\end{array}$

Languages of Oceania and Australasia (Austronesian, Papuan, and Australian), A-Z

BS335.D87 Dupaninan Agta TABLE BS5

\section{The Bible}

Old Testament

Works about the Old Testament

Topics (not otherwise provided for), A-Z

CLASS BT

$$
\begin{aligned}
& \text { Doctrinal theology } \\
& \text { History of specific doctrines and movements. Heresies and schisms } \\
& \text { By period } \\
& \text { Modern, 1517- }
\end{aligned}
$$

BT1477
CLASS BV

Practical theology

Missions

Missions in individual countrie

By region or country

BV3625.S68-S682 $\quad$ South Sudan or countries, A-Z

CLASS BX

Christian denominations

Catholic Church

Monasticism. Religious orders

Individual orders of men
BX3479 Congregation of the Holy Family of Nazareth TABLE BX18

graphy and portraits

Individual

BX4705.P3786 Patachich, Gábor

Christian denominations

ther Protestant denominations

Baptists

BX6329.P46

\section{Methodism}

Individual branches of Methodists

United Methodist Church (United States). Methodist Church (United States). Methodist Episcopal Church

BX8385.S49 Special topics, A-Z

$$
\text { Sex }
$$

Mormons. Church of Jesus Christ of Latter-Day Saints Special topics
Other, A-Z

BX8643.R33 Race relations

BX8643.W45

Well-being

Pentecostal churches

Individual branches

True Jesus Church TABLE BX3
Thivial branches 
Shakers. United Society of Believers. Millennial Church

Other topics, A-Z

BX9789.A47

$$
\text { Aesthetics }
$$

CLASS DS

Israel (Palestine). The Jews

Jerusalem

Description. Antiquities and exploratio

Other special places and objects, A-Z

DS109.8.G48$$
\text { Geula }
$$

CLASS HQ

\section{The family. Marriage. Home}

Family size

Family planning. Birth control

Abortion

Religious aspects

dividual religions and denominations

HQ767.36 Russian

CLASS KBU

Law of the Roman Catholic Church. The Holy See Local Church governmen

By region
Africa

KBU4589.5 Eswatini (2018-) TABLE KB4

CLASS N

$$
\begin{aligned}
& \text { Visual arts } \\
& \text { Special subjects of art } \\
& \text { Religious art } \\
& \text { Christian art } \\
& \text { Special subjects } \\
& \text { Apostles. Saints } \\
& \text { Special apostles or saints, A-Z }
\end{aligned}
$$

N8080.T47
CLASS P

Philology. Linguistics

Communication. Mass media

$$
\text { Special aspects }
$$

Relation to special groups of people, A-Z

$\begin{array}{ll}\text { [P94.5.F332] } & \text { Faith healers see P94.5.H43-.H432 } \\ \text { P94.5.H43-.H432 } & \text { Healers TABLE P1 }\end{array}$

In faith healers, spiritual healers, etc.

Spiritual healers see P94.5.H43-H432

P96.H42-H422

Other, A-Z

96.H42-H422)

Healers

Including faith healers, spiritual healers, etc. CANCEL see P94.5 $443-4432$

CLASS PG

Slavic. Baltic. Albanian

$$
\begin{aligned}
& \text { Russian literature } \\
& \text { Collections of Russian literature } \\
& \text { Special topics, A-Z }
\end{aligned}
$$

PG3205.A84

$$
\text { Atheism }
$$

CLASS PK

Indo-Iranian philology and literature

Modern Indo-Aryan languages

Particular languages and dialects, $A-Z$

Bengali Literature Individual authors

Through 1960

Tagore, Rabindranath, 1861-1941
Criticism

PK1727.M88

$$
\begin{aligned}
& \text { Special topics, A-Z } \\
& \text { Muslims }
\end{aligned}
$$

CLASS PN

Literature (General)

Theory. Philosophy. Esthetics

Relation to and treatment of special elements, problems, and subjects Other special

PN56.P58 


\section{CLASS Z}

Libraries

ibrary science. Information science

Information organization

$$
\text { Metadata }
$$

$[\mathrm{Z} 666.7-73]$

$$
\text { Cf. ZA3274 Metadata theory (Information resources) }
$$

\section{The collections. The books}

Special classes of materials

Z692.D37

$$
\begin{aligned}
& \text { Other classes, A-Z } \\
& \text { Data sets }
\end{aligned}
$$

CLASS ZA

ZA3073

formation resources (General)

Information ethics. Moral and ethical aspects of information use

[ZA3075-3080] Research. Seeking and finding information. Information retrieval CANCEL

[ZA3075-3080] Seeking and finding information. Information retrieval Including information behavior and information literacy CANCEL Including information behavio

ZA3075

Research. Seeking and finding information. Information retrieval General works CANCEL

Seeking and finding information. Information retrieval General works

ZA3075

Research. Seeking and finding information. Information retrieval Juvenile works CANCEL

ZA3080

Seeking and finding information. Information retrieval

ZA3080

$$
\text { Juvenile works }
$$

[ZA3083-308

esearch. Seeking and finding information. Information retrieval Information filtering systems CANCEL

Seeking and finding information. Information retrieval Information filtering systems

Research. Seeking and finding information. Information retrieval Information filtering systems

ZA3083
ZA3083

ZA3084

ZA3084

ZA3085

ZA3085

ZA3085.5

[ZA3088-3090]

ZA3088

ZA3088.5.A-Z

ZA3088.5.C65

ZA3088.5.S34

ZA3273-3274

ZA3273

ZA3274

[ZA3274]
Seeking and finding information. Information retrieval

Information filtering systems

General works

Research. Seeking and finding information. Information retrieval formation filtering systems Recommender systems CANCEL

Seeking and finding information. Information retrieval Information filtering systems Recommender systems

Research. Seeking and finding information. Information retrieval Information filtering system

Filter bubbles CANCEL

Seeking and finding information. Information retrieval Information filtering systems

$$
\text { Filter bubbles }
$$

nformation resources management. Knowledge management Including information overload

ion literacy

General

Special groups, A-Z

College students

High school students

Information organization

For information organization in general see Z666.5

General works

Metadata

$$
\text { Class here metadata theory in general }
$$

Cf 7666.7-73 Metadata in libraries 


\section{TABLES}

\section{TABLE BX16}

Table for individual Catholic religious orders (56 numbers)

History

By region or country

Europe

Other European regions or countries, A-Z

BX16 45.B28 Balkan Peninsula

TABLE Z1

Z1 14.F37

National bibliography. Imprints

Literature (General) Special topics, A-Z

Fasts and feasts 